\title{
Perfil de competencias del profesional que cumple funciones de monitoreo, evaluación y gestión de evidencias de programas y proyectos de desarrollo. Un aporte para las decisiones basadas en evidencias
}

\author{
Monitoring, evaluation and evidence-based management in \\ development projects: professional competency profile. \\ A contribution for evidence-based decision making
}

Eva Miranda ${ }^{1}$, Adriana Torres ${ }^{1}$, Emma Rotondo $^{1}$, Patricia Mostajo ${ }^{1}$

Proyecto Evaluations, contratista de USAID

\begin{abstract}
Resumen
Los programas y proyectos de desarrollo que forman parte de las políticas públicas necesitan ser eficientes en la inversión y eficaces en el logro de sus objetivos y resultados. El monitoreo y la evaluación (ME) son funciones estratégicas y sistemáticas que buscan generar información confiable y rigurosa acerca del alcance y logros de metas y resultados previamente establecidos en los prog ramas y proyectos de desarrollo. Existen consensos internacionales sobre la necesidad de desarrollar capacidades y profesionalizar el ME, adoptar estándares comunes, y reforzar las estrategias de desarrollo nacional, mejorando el uso de las evaluaciones para retroalimentar las políticas públicas y lograr evidencias que sustenten un desarrollo sostenible con inclusión y equidad. En nuestro pais, los programas sociales y diversos programas presupuestales que desarrolla el Estado peruano, así como los programas y proyectos que apoya la cooperación internacional, hacen evidente la demanda de sistemas nacionales de ME capaces de obtener evidencias rigurosas sobre los resultados y que estos se reflejen tanto en la asignación presupuestal, como en la mejora de procesos y estrategias. El perfil de competencias del profesional que cumple funciones de ME es el conjunto de conocimientos, habilidades y actitudes que debe poseer la persona para desempeñar adecuadamente sus funciones. En el presente artículo se identifican las funciones de los profesionales en el área de ME y se propone el perfil de competencias que deben adquirir así como los contenidos de las competencias específicas de ME. Se presenta el perfil de competencias validado como un aporte a la gestión por resultados y decisiones basadas en evidencias para su implementación en procesos de gestión de recursos humanos y sistemas de ME; es una herramienta para ser aplicada prioritariamente en la planificación y dotación, organización del trabajo, el desarrollo de capacidades y la compensación en el proceso de gestión de recursos humanos en el área y sistemas de ME. El perfil de competencias del profesional que cumple funciones de ME constituye una experiencia pionera en el país, y se pone a disposición de las instituciones académicas, instituciones gubernamentales $u$ otras que ofrecen servicios de ME y a la comunidad en general.
\end{abstract}

Palabras clave: Políticas públicas, evaluación, monitoreo.

\section{Abstract}

Programs and development projects that are part of public policies need to be efficient in investment and effective in achieving objectives and results. Monitoring and evaluation (ME) are strategic and systematic functions that seek to generate rigorous and reliable information on effects of programs and development projects. There is consensus about the need to develop capacity and professionalize ME, to adopt common standards, and to strengthen national development strategies. This should result in better public policies, evaluations, which should support sustainable development with equity and inclusion. In our country, public social and budget programs, as well as programs and projects funded by international agencies require ME systems to obtain rigorous evidence on outcomes. They should be reflected in both budget allocation and in the improvement of processes and strategies. This article identifies specific functions of ME professionals, and proposes a profile of skills, attitudes and knowledges and specific competencies. A validated profile is presented as a contribution for results-based management and evidence-based decision making, and for its implementation in processes and systems related to human resources and ME management. It is a tool to be used primarily in planning and provision, work organization, capacity development and compensation. It is a pioneering experience in the country, and it is available for academic and governmental institutions or other actors involved in ME services.

Keywords: Public policies, evaluation, monitoring.

An Fac Med. 2015;76:67-76 / doi:10.15381/anales.v76i1.10974 


\section{INTRODUCCIÓN}

Los programas y proyectos de desarrollo que forman parte de las políticas públicas contienen una serie de estrategias $\mathrm{y}$ acciones que tienen como finalidad mejorar las condiciones y calidad de vida de una población dándoles oportunidades para lograr equidad en el acceso a servicios e inclusión económica y social sostenible. Para ello es necesario que sean eficientes en la inversión y eficaces en el logro de sus objetivos y resultados. El monitoreo y la evaluación (ME) son funciones estratégicas y sistemáticas que buscan generar información confiable y rigurosa acerca del alcance y logros de metas y resultados previamente establecidos; el propósito de ME es verificar y medir los avances y logros en los resultados previstos y no previstos de los programas, e identificar los problemas y mejorar la gestión y desempeño así como las decisiones de los gestores. ${ }^{(1)}$

La necesidad de implementar estándares comunes de ME surge desde la década de los setenta con la Asociación Americana de Evaluación y en las décadas siguientes mediante diversos organismos internacionales y asociaciones profesionales de evaluación; sin embargo, el acuerdo internacional sobre la necesidad de desarrollar capacidades y profesionalizar el ME, adoptar estándares comunes y fortalecer la capacidad nacional gubernamental surge con énfasis en los siguientes consensos: Declaración de París sobre la Eficacia de la Ayuda al Desarrollo (2005), el Programa de Acción de Accra (2008), y la Declaración de Busan (2011) ${ }^{(1,2)}$. Estos consensos precisan que, en el marco de los Objetivos de Desarrollo del Milenio, es fundamental reforzar las estrategias de desarrollo nacional de los países socios, mejorando el uso de las evaluaciones para retroalimentar las políticas públicas y lograr evidencias que sustenten un desarrollo sostenible con inclusión y equidad. Uno de los organismos que impulsó la aprobación y uso de los estándares para la evaluación como incentivo para la mejora de las prácticas evaluativas es el Comité de Ayuda al Desarrollo (CAD) de la Organización de Cooperación y Desarrollo Económico (OCDE) ${ }^{(3)}$.

Recientemente, en el año 2012, se conformó EvalPartners, un movimiento global para el fortalecimiento de las capacidades nacionales de evaluación. Dicha iniciativa involucra a las oficinas de evaluación de las organizaciones de las Naciones Unidas, agencias bilaterales como USAID así como a Organizaciones Voluntarias de Evaluación. EvalPartners ha declarado que el año 2015 será el Año Internacional de la Evaluación. Como consecuencia de este y otros esfuerzos, en los últimos años se ha manifestado un interés, voluntad política y asignación de recursos para desarrollar capacidades nacionales en ME, lo cual constituye una oportunidad para todas las instituciones que trabajan en el tema ${ }^{(4)}$.

En nuestro país, los programas sociales y diversos programas presupuestales que desarrolla el Estado peruano, así como los programas y proyectos que apoya la cooperación internacional, entre otros, hacen evidente la demanda de sistemas nacionales de ME capaces de obtener evidencias rigurosas sobre los resultados y que estos se reflejen tanto en la asignación presupuestal como en la mejora de procesos y estrategias. Actualmente dichos programas están centrados en la elaboración de información sobre productos y en el monitoreo del alcance a beneficiarios -con escaso uso de esta información- más que en la evaluación de resultados, o sea de los cambios en el mediano y largo plazos.

Estudios previos señalan que los sistemas de $\mathrm{ME}$ internos funcionan mejor si cuentan con una planificación orientada a resultados (productos, efectos e impactos), lo que significa un marco operacional con roles y delegaciones para el ME, recursos humanos y financieros, así como trabajo en equipo y motivación en un proceso participativo que lleve al uso de la información para tomar decisiones ${ }^{(5)}$. $\mathrm{Al}$ respecto, el año 2014 el Ministerio de Economía y Fi- nanzas (MEF) solicitó un diagnóstico de los actuales mecanismos de seguimiento y evaluación que se aplican en el Perú; entre los resultados se evidencian una débil gestión del conocimiento e insuficiente nivel de capacidades técnicas para desarrollar actividades de seguimiento y evaluación ${ }^{(6)}$. Asimismo, el Ministerio de Desarrollo e Inclusión Social (MIDIS) señaló que "Tradicionalmente los sistemas de ME se han concentrado en recopilar y producir información, pese a que las evidencias y el conocimiento que provienen de las revisiones sistemáticas, metaanálisis y evaluaciones, deben constituir la base para la toma de decisiones para el diseño de nuevos programas, la asignación presupuestal; la formulación de metas de cobertura, focalización y desempeño; el rediseño, restructuración/modificación o cierre de programas sociales, así como para la priorización de ámbitos geográficos de intervenciones efectivas y de proyectos de inversión" (7).

En el Perú y en la región de América Latina no existe la certificación profesional por competencias de monitores y evaluadores. Existen algunos perfiles de competencias pero no profundizan lo relacionado a la evaluación de políticas, programas y proyectos de desarrollo; por consiguiente, se encuentran limitaciones en la implementación y desarrollo de los procesos de gestión de recursos humanos de estas áreas (especialmente en la planificación y provisión, organización del trabajo, desarrollo de capacidades y aplicación de mecanismos de compensación). Esto generó la necesidad de impulsar la identificación de las competencias como una contribución al cumplimiento de las funciones de ME que las instituciones demandan. Si no conocemos y desarrollamos las competencias de los profesionales que cumplen funciones de ME será difícil implementar o construir sistemas de evaluación y monitoreo. La presente propuesta, generada por el Proyecto Evaluations, abre un camino para orientar las funciones de ME y afianzar el desarrollo de capacidades en base a competencias. 
Mención aparte, el abordaje de la gestión de recursos humanos por competencias no es nuevo en el país. Específicamente, el Instituto de Desarrollo de Recursos Humanos del Ministerio de Salud, a partir de experiencias nacionales y regionales del país, publicó el año 2005 la metodología para la formulación de perfiles de competencias para trabajadores del primer nivel de atención. Posteriormente, el año 2011 publicó las competencias laborales para la mejora del desempeño de los recursos humanos en salud, incluyendo las competencias genéricas para los trabajadores del sector y las competencias laborales específicas para el personal de salud del primer nivel de atención ${ }^{(8)}$.

La certificación profesional por competencias se institucionalizó en nuestro país el año 2007 con la aprobación del Reglamento de la Ley № 28740, Ley del Sistema Nacional de Evaluación, Acreditación y Certificación de la Calidad Educativa (SINEACE). Con ello se dio inicio a la certificación de competencias laborales y profesionales, contándose a la fecha con nueve entidades certificadoras de competencias autorizadas por el Consejo de Evaluación, Acreditación y Certificación de la Calidad Educativa de la Educación Universitaria (CONEAU); estos son los colegios profesionales: médico, de enfermeros, de obstetras, odontológico, farmacéutico, de nutricionistas, de psicólogos, de biólogos y tecnólogos médicos. Cada entidad certificadora cuenta con los mapas de competencia profesionales y sus respectivos estándares de competencia ${ }^{(9)}$. Otro hecho importante fue la aprobación, el año 2010, del Plan Nacional de Desarrollo de Capacidades para la Gestión Pública y Buen Gobierno de los Gobiernos Regionales y Locales, cuyo enfoque educativo es por competencias ${ }^{(10)}$. Asimismo, el Decreto Supremo No. 009-2010-PCM y su Reglamento No. 1025 sobre las normas de capacitación y rendimiento para el sector público, entre otros aspectos, establece que las actividades de capacitación y evaluación de las personas al servicio de Estado de cada unidad pu- blica se realice mediante la elaboración del Plan de Desarrollo de las Personas (PDP), siendo uno de sus contenidos mínimos las competencias necesarias de las personas para alcanzar los objetivos estratégicos. Los PDP deben ser presentados a la Autoridad Nacional del Servicio Civil (SERVIR) y ejecutarlos de acuerdo a su cronograma establecido ${ }^{(11)}$. En síntesis, en la actualidad, el enfoque de competencias es empleado por las instancias gubernamentales encargadas de la calidad educativa como el SINEACE y sus órganos operadores, y se aplican en la formación de los recursos humanos y en los procesos de certificación profesional periódica; es empleado también por SERVIR, en la normatividad general del sistema de recursos humanos.

\section{ASPECTOS CONCEPTUALES}

El perfil de competencias del profesional que cumple funciones de ME es el conjunto de rasgos (conocimientos, habilidades y actitudes) que debe poseer la persona para desempeñar adecuadamente sus funciones. Se constituye en un modelo conciso que describe comportamientos que se pueden advertir en el desempeño.

El perfil de competencias es un modelo integral que describe comportamientos que se pueden observar, desarrollar y evaluar en el desempeño; permite introducir el enfoque de competencias en una propuesta de desarrollo de capacidades, favoreciendo el desarrollo integral de la persona; favorece la estandarización (uso de un lenguaje común) y la articulación entre el mundo laboral y educativo; mejora la empleabilidad y la adaptación del profesional a espacios laborales cambiantes; y favorece el involucramiento de capital humano competitivo en el desarrollo de un sistema de ME que el país necesita.

Para el presente trabajo, se ha optado por la definición de competencias profesionales de la Organización Internacional del Trabajo (OIT), que la define como la idoneidad para desempeñar una tarea o desempeñar un puesto eficazmente, por poseer las calificaciones requeridas para ello. El convenio 195 de recomendación sobre el desarrollo de los recursos humanos -educación, formación y aprendizaje permanente - a la letra señala "el término competencia abarca los conocimientos, las aptitudes profesionales y el saber hacer que se dominan y aplican en un contexto específico" ${ }^{(12)}$

En ese marco, el perfil abarca dos tipos de competencia:

- Las competencias específicas que son las competencias técnicas que aplica el monitor y/o evaluador en el desempeño de sus funciones especializadas. Están vinculadas a la parte técnica de los procesos que abarca el ME.

- Las competencias claves, llamadas también centrales o genéricas. Estas tienen conexión con las características de tipo personal social de la persona y facilitan la adaptación, la sociabilidad y la organización para el trabajo. Tienen que ver con las habilidades sociales, la capacidad para trabajar en equipo, la actualización permanente para adaptarse a los cambios, la conducta ética, entre otras ${ }^{(13)}$.

Se utilizó el análisis funcional para la identificación de las competencias inherentes a una profesión, ocupación, institución, organización o servicio. Se inició identificando el propósito principal del sujeto de análisis (en este caso el profesional que cumple funciones de ME) para enunciar y correlacionar sus funciones hasta llegar a especificar las competencias que debe lograr y que le permiten la realización exitosa de una actividad. Adicionalmente, hemos propuesto los contenidos para cada competencia específica; es decir, los componentes principales o saberes compuestos por conocimientos (saber), habilidades (saber hacer) y actitudes (saber ser). 


\section{OBJETIVOS}

Los objetivos estuvieron centrados en la identificación y validación de las competencias del profesional que cumple funciones en las áreas de monitoreo, evaluación y gestión de evidencias, como sigue:

- Identificar las funciones generales que cumplen los profesionales de estas áreas.

- Proponer las competencias específicas y claves o personal-sociales que debe lograr todo profesional que ejecuta estas funciones.

- Proponer los contenidos principales de las competencias específicas de ME.

\section{METODOLOGÍA APLICADA EN LA CONSTRUCCIÓN DEL PERFIL DE COMPETENCIAS}

Antes de iniciar la construcción del mapa de competencias se revisó la base teórica, la búsqueda de antecedentes y entrevistas a expertos sobre el tema. No se encontraron perfiles específicos de ME elaborados con el análisis funcional; sin embargo, existen experiencias de aplicación del método en diferentes áreas profesionales y ocupacionales e información relevante que contribuyeron a la construcción de la propuesta.

Se revisaron documentos normativos del MEF (Ley de Presupuesto donde algunos artículos explicitan actividades de seguimiento y evaluación), del MIDIS (Lineamientos para el seguimiento, evaluación y gestión de la evidencia), del Ministerio de la Mujer y Poblaciones Vulnerables (MIMP) (Lineamientos para el monitoreo y evaluación sectorial), de SERVIR (perfiles específicos por tipo de puesto del Cuerpo de Gerentes Públicos) y los perfiles que demandan las instituciones para contratar al personal para trabajos relacionados con el ME de programas sociales.

Asimismo, se revisaron publicaciones del MINSA (competencias laborales para la mejora del desempeño de los recursos humanos en salud) y programas académicos tales como el plan curricular de la Diplomatura de Especialización en Monitoreo y Evaluación de Proyectos y Programas Sociales de la Pontificia Universidad Católica del Perú y el plan curricular del Diplomado de Gestión de la Información de las Intervenciones Sanitarias del MINSA, dirigido por la Dirección General de Gestión y Desarrollo de Recursos Humanos en Salud y el Programa de Apoyo a la Reforma del Sector Salud II; este último aplicó el análisis funcional y presenta como una de sus funciones el ME de la implementación de intervenciones sanitarias ${ }^{(14)}$.

A nivel internacional se revisaron las competencias centrales del evaluador propuesto por la Red de Seguimiento, Evaluación y Sistematización en América Latina y el Caribe (RELAC), el documento Evaluator Competencies (International Board of Standards for Training, Performance and Instruction), el documento Evaluation Capabilities Framework (United Kingdom Evaluation Society), el documento Competencies for Development Evaluators Managers and Commissioners (International Development Evaluation Association), el Program Evaluation Forms and Approaches (John Owen y Patricia Rogers), los estándares de evaluación (CAD), el diagnóstico de funcionamiento de los sistemas de seguimiento evaluación y medición del desarrollo de capacidades (PREVAL) y el documento Evaluation Policy (USAID).

Tabla 1. Funciones del profesional de monitoreo y evaluación (ME) según áreas de competencia.

\begin{tabular}{ll}
$\begin{array}{l}\text { ÁREAS DE COMPETENCIA } \\
\text { (Áreas básicas de la transdisciplina) }\end{array}$ & $\begin{array}{l}\text { FUNCIONES } \\
\text { ¿Qué debe hacer? }\end{array}$ \\
& $\begin{array}{l}\text { Diseñar el plan de ME de programas y proyectos de desarrollo, } \\
\text { considerando los resultados esperados y el momento en el } \\
\text { ciclo del programa (anual, multianual). } \\
\text { GONITOREO } \\
\text { Gestionar el plan de ME con flexibilidad y rigurosidad técnica } \\
\text { usando las tecnologías de información y comunicación. }\end{array}$ \\
\hline EVALUACIÓN & Solicitar y/o diseñar evaluaciones según tipo y técnicas. \\
& Desarrollar evaluaciones según tipo y técnicas. \\
\hline GESTIÓN DE LA EVIDENCIA & $\begin{array}{l}\text { Gestionar evidencias provenientes del ME, para promover la } \\
\text { rendición de cuentas y el aprendizaje. }\end{array}$ \\
\hline
\end{tabular}

Para proponer las competencias se determinaron tres líneas orientadoras, dos de ellas previamente establecidas por USAID, que permitieron identificar dos tipos de competencias: específicas y claves o personal-sociales. Las líneas orientadoras fueron:

- El trabajo con enfoque en los resultados, el aprendizaje y la toma de decisiones basadas en la evidencia ${ }^{(15)}$. Esta línea orientó la identificación de las competencias específicas para el monitoreo, diseño y desarrollo de evaluaciones; asimismo, para la gestión de los resultados y evidencias según estándares internacionales.

- Práctica del monitor y evaluador en contextos de transparencia y participación ciudadana ${ }^{(15)}$. La línea fue el referente para la propuesta de las competencias claves o personal-sociales dirigidas a la visión de conjunto, alianzas, innovación, promoción de la cultura del aprendizaje de la evaluación, la conducta ética y moral, la educación continua y habilidades sociales.

- Competencias dirigidas al cumplimiento de funciones, para lo cual se eligió el método del análisis funcional.

El proceso se inició con la propuesta del propósito principal que los profesionales del ME deben alcanzar en el puesto o función, siendo el siguiente: generar evidencias y contribuir al uso de las mismas en la formulación y eje- 
cución de políticas, planes, proyectos y programas de desarrollo. Se identificaron cinco funciones que los profesionales deben cumplir para el logro del propósito principal, según áreas de la transdisciplina: monitoreo, evaluación y gestión de la evidencia (tabla 1).

El proceso continuó con la identificación de las competencias que debe tener el profesional que cumple funciones de ME para desempeñar con éxito estas funciones, resultando el mapa de competencias del profesional que cumple funciones en ME (Anexo 1). Dicho mapa de competencias incluye las competencias específicas para el diseño y gestión del monitoreo, diseño y desarrollo de evaluaciones, y gestión de evidencias. La identificación de las competencias específicas, descritas en el mapa, se realizó con el método del análisis funcional descrito en la figura 1.

También se plantearon las competencias claves a través del análisis documental, entrevista a expertos y revisión de la literatura internacional, resultando siete competencias para el monitor - evaluador: Facilitación de procesos, Desarrollo profesional y autodesarrollo del talento, Escucha activa, Comportamiento guiado por valores y principios éticos, Práctica de valores democráticos y participativos, Negociación, y Liderazgo de equipos.
Finalmente, las competencias específicas antes de su aplicación fueron validadas a través del juicio de expertos con la participación de especialistas en ME de la PUCP, del MIDIS, el MIMP, Ministerio del Ambiente, del Banco Mundial, ConsultAndes, USAID, proyectos de USAID: Políticas en Salud y Evaluations, ONUSIDA, la Red Peruana de Evaluación (Red EvalPerú), la Red Peruana de Monitoreo y Evaluación (Red PERUME), la Red de Seguimiento Evaluación y sistematización en América Latina y El Caribe (RELAC) y consultores privados en evaluación que se desempeñan en las áreas de salud, ciencias sociales y economía. Los especialistas consultados revisaron, corrigieron, complementaron o ratificaron cada competencia específica aplicando los siguientes criterios: pertinencia, verificación del desempeño, claridad de redacción, incorporación de notas complementarias.

Un valor agregado del proceso de validación de las competencias fue que los especialistas en ME incorporaron a cada competencia específica sus contenidos principales que forman parte de ella: conocimientos (saber), habilidades (saber hacer) y actitudes (saber ser), lo cual tendrá diversas aplicaciones tales como normalizar la competencia (condiciones en que la persona debe mostrar su aptitud), elaborar instrumentos

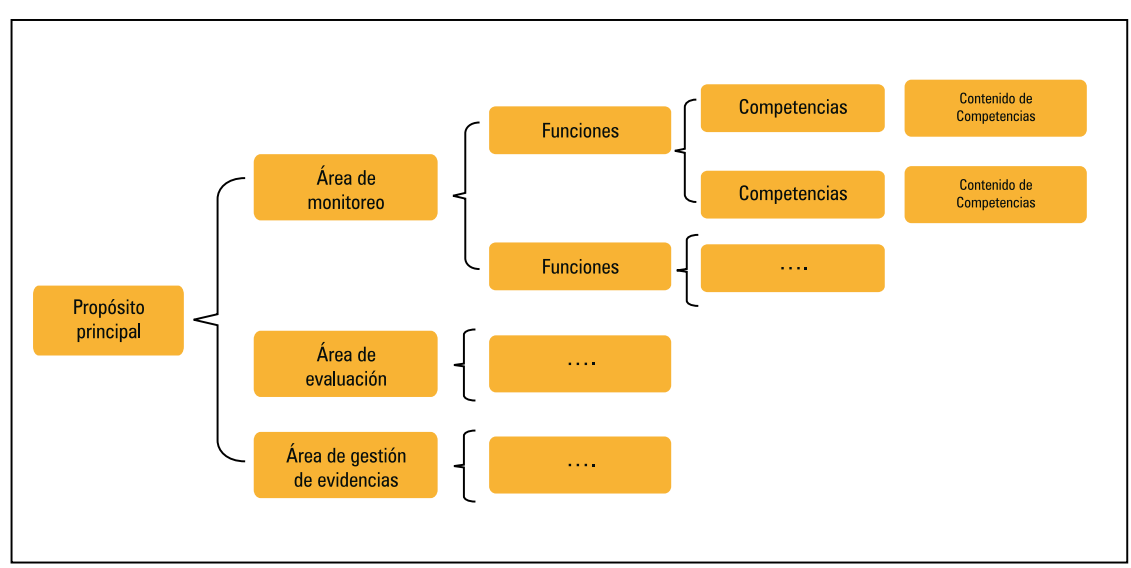

Figura 1. Ruta seguida para la identificación de competencias específicas en monitoreo, evaluación, gestión de evidencias, y sus contenidos de evaluación de competencias, planificar y desarrollar programas de desarrollo de capacidades de las personas, entre otras.

Culminado este proceso, se inició una primera aplicación para el autodiagnóstico de necesidades de fortalecimiento de competencias a integrantes de redes de evaluación del país, lo cual permitió hacer mejoras a la propuesta.

\section{PERFIL DE COMPETENCIAS}

Se presenta el perfil de competencias validado como un aporte a la gestión por resultados y decisiones basadas en evidencias para su implementación en procesos de gestión de recursos humanos y sistemas de ME.

El logro de las competencias específicas en un profesional será gradual y de acuerdo a las funciones que cumple. Implica desarrollar conocimientos, habilidades y actitudes que generan diferentes procesos, algunos más complejos que otros, hasta lograrlo; por ello, será necesario trabajar con el enfoque de equipo buscando la complementariedad entre sus integrantes (garantizar que en el equipo existan integrantes con las diferentes competencias) en tanto cada integrante va logrando el conjunto de competencias. Esto no aplica a las competencias claves, porque son transversales y que todo profesional del área de ME debe lograr.

\section{APLICACIONES INICIALES}

Las competencias para evaluación, monitoreo y gestión de los planes, programas y proyectos de desarrollo han tenido las siguientes aplicaciones:

- La elaboración de un instrumento de autodiagnóstico de necesidades de fortalecimiento de competencias del personal que trabaja en las áreas de $\mathrm{ME}$ con su respectivo aplicativo informático.

- Dos redes de evaluación y doce instituciones del país (sector público 
Tabla 2. Competencias específicas para el Monitoreo de planes, programas y proyectos de desarrollo.

\begin{tabular}{|c|c|}
\hline $\begin{array}{l}\text { ÁREA DE } \\
\text { COMPETENCIA }\end{array}$ & COMPETENCIAS \\
\hline MONITOREO & $\begin{array}{l}\text { Diseñar el plan de monitoreo y evaluación en función a los objetivos que se espera alcanzar con el Plan / Programa / Proyecto / Política. } \\
\text { Diseñar los instrumentos / herramientas de recojo de datos en función al plan de monitoreo y evaluación. } \\
\text { Organizar el proceso de recolección de datos en función al instrumento de recolección. } \\
\text { Recolectar datos utilizando instrumentos/herramientas de recojo de información acorde con los indicadores establecidos. } \\
\text { Verificar la calidad del dato recolectado de acuerdo a los criterios preestablecidos. } \\
\text { Procesar los datos en función de las necesidades de análisis. } \\
\text { Organizar la información en función a los objetivos del Plan / Programa / Proyecto / Política. } \\
\text { Elaborar reportes de monitoreo proporcionando recomendaciones según los diversos usuarios. }\end{array}$ \\
\hline
\end{tabular}

Tabla 3. Competencias específicas para la Evaluación de planes, programas y proyectos de desarrollo.

\begin{tabular}{l|l}
$\begin{array}{l}\text { ÁREA DE } \\
\text { COMPETENCIA }\end{array}$ & COMPETENCIAS \\
& $\begin{array}{l}\text { Diseñar el plan de evaluaciones de acuerdo a las necesidades y usuarios del Plan/ Programa / Proyecto / Política. } \\
\text { Elaborar términos de referencias acorde al diseño de evaluación requerida. } \\
\text { Elaborar el diseño de las evaluaciones según tipo (ex ante, líneas de base, desempeño, efecto e impacto) y técnicas (cualitativas, } \\
\text { cuantitativas o mixtas) de evaluación. } \\
\text { Elaborar instrumentos de recojo de información válidos y confiables según el diseño de la evaluación. } \\
\text { Recolectar la información aplicando técnicas apropiadas con estándares de control y calidad. }\end{array}$ \\
& $\begin{array}{l}\text { Procesar la información verificando la calidad de la data. } \\
\text { Categorizar la información de la evaluación de acuerdo a las preguntas, objetivos y tipo de evaluación. } \\
\text { Identificar los hallazgos significativos en función a la pregunta y objetivos de la evaluación. } \\
\text { Redactar las conclusiones sintetizando y comparando las evidencias con juicios de valor y mérito. } \\
\text { Formular recomendaciones de acuerdo al contexto institucional, a los objetivos de evaluación, incorporando la perspectiva de los usuarios. } \\
\text { Formular lecciones aprendidas de acuerdo a los resultados de la evaluación. } \\
\text { Elaborar el informe final de la evaluación según estándares adoptados por la institución. } \\
\text { Evaluar el diseño y el informe final de una evaluación aplicando estándares adoptados por la institución. }\end{array}$ \\
\hline
\end{tabular}

Tabla 4. Competencias específicas para la Gestión de evidencias de planes, programas y proyectos de desarrollo.

\begin{tabular}{|c|c|}
\hline $\begin{array}{l}\text { ÁREAS DE } \\
\text { COMPETENCIA }\end{array}$ & COMPETENCIAS \\
\hline $\begin{array}{l}\text { GESTIÓN DE LA } \\
\text { EVIDENCIA }\end{array}$ & $\begin{array}{l}\text { Gestionar la implementación de las recomendaciones del monitoreo de acuerdo a los objetivos del Plan / Programa / Proyecto / Política. } \\
\text { Diseñar un plan de uso de las recomendaciones de la evaluación según usuarios } \\
\text { Elaborar el plan de uso y gestión de evidencias, según usuarios. } \\
\text { Identificar evidencias aplicando herramientas ad hoc, para la toma de decisiones. } \\
\text { Aplicar estrategias de facilitación y comunicación de resultados y de evidencias utilizando tecnologías de información y comunicación según } \\
\text { usuarios. }\end{array}$ \\
\hline
\end{tabular}

Tabla 5. Competencias claves (personal-sociales) ${ }^{(16-18)}$.

\section{Competencias claves - Personal-sociales}

Facilitación de procesos: Capacidad para acompañar y propiciar espacios para el aprendizaje significativo (útil para el cambio).

Desarrollo profesional y autodesarrollo del talento: Búsqueda del aprendizaje continuo, mantenerse actualizado y poder incorporar nuevos conocimientos a su área de trabajo para obtener mejores resultados.

Escucha activa: Capacidad de entender el juicio del otro con respeto y apertura.

Comportamiento guiado por valores y principios éticos: Capacidad de sentir y obrar en todo momento de acuerdo con los valores morales, respetando a las personas y políticas organizacionales.

Práctica de valores democráticos y participativos: Capacidad para aplicar enfoques y perspectivas incluyentes y no discriminatorias.

Negociación: Capacidad para persuadir a otras personas, utilizando argumentos honestos y basados en evidencias, acercando posiciones de las partes interesadas, para construir acuerdos guiados por el concepto de ganar-ganar.

Liderazgo de equipos: Capacidad para asegurar una adecuada conducción de personas, desarrollar el talento, lograr y mantener un clima de trabajo armónico. 
nacional y regional y ONGs) han aplicado el instrumento y cuentan con el diagnóstico de necesidades de fortalecimiento de competencias en ME.

- Los resultados en las redes de evaluación revelan necesidades de fortalecimiento de competencias diferenciadas; sin embargo, el promedio para el diseño y desarrollo de evaluaciones se ubica en el nivel de necesidad de 'refuerzo y actualización'. Cabe anotar una mayor necesidad de fortalecimiento en las competencias para la gestión y uso de evidencias.

- En las instituciones del sector público y ONGs, los resultados evidencian una necesidad de fortalecimiento de competencias para el desempeño de la función de monitoreo a nivel de 'refuerzo y actualización', ubicándose los valores obtenidos más cerca al nivel inferior 'desarrollo desde lo básico' que al nivel superior 'nivel avanzado'. La necesidad de fortalecimiento de competencias para el desempeño de la función de evaluación es mayor en relación a la de monitoreo; es así que los encuestados necesitan un nivel de fortalecimiento de competencias que requiere 'desarrollarse desde lo básico'.

- Se ha elaborado un programa de posgrado para el fortalecimiento de competencias en ME que será desarrollado los años 2015 y 2016.

\section{CONCLUSIONES}

El perfil de competencias del profesional que cumple funciones de monitoreo y evaluación constituye una experiencia pionera en el país, y permite poner a disposición de las instituciones académicas, instituciones gubernamentales u otras que ofrecen servicios de $\mathrm{ME}$ y a la comunidad en general, las competencias que debe tener un profesional que trabaja en el área de ME según corresponda.
La consideramos como una herramienta que puede aplicarse en el proceso de gestión de recursos humanos del área de ME desde la formación, con énfasis en la planificación y provisión (reclutamiento y selección de personal), la organización del trabajo (inducción, diseño de ocupaciones, evaluación del desempeño), el desarrollo (entrenamiento, formación, capacitación, especialización) y la compensación (planes de incentivos al buen desempeño). Asimismo, es una herramienta para el autodesarrollo profesional, de manera que cada evaluador conozca sus niveles y busque incrementar sus competencias a lo largo de su vida profesional.

Es un aporte que contribuye al posicionamiento de la cultura y práctica del monitoreo y la evaluación como un proceso fundamental, social y político, dentro de un contexto de mayor transparencia y participación ciudadana.

\section{REFERENCIAS BIBLIOGRÁFICAS}

1. Maldonado C, Galíndez C, editores. Monitoreo, Evaluación y Gestión por Resultados. $1^{\text {a }}$ Edición. México: CIDE Centro CLEAR para América Latina; 2013.

2. Bena F, Las claves de Busan [Nota informativa de Oxfam]. Reino Unido: Oxfam Internacional; 2012 [Consultado: 29-12-13]. Disponible en: http://www. oxfam.org/sites/www.oxfam.org/files/bn-busannutshell-global-partnership-021012-es.pdf.

3. Organización de Cooperación y Desarrollo Económicos. Estándares de Calidad para la Evaluación del Desarrollo. España: Ministerio de Asuntos Exteriores y de Cooperación; 2010.

4. EvalPartners [homepage en internet]. EE.UU. Evidence for the world we want; c2013 [Consultado: 29-12-13]. Disponible en: http://mymande. org/evalyear/Declaring_2015_as_the_International_Year_of Evaluation.

5. Ccori D. Institucionalización de los sistemas de monitoreo y evaluación de lucha contra la pobreza rural. Lima: Plataforma Regional de Desarrollo de Capacidades en Evaluación y Sistematización en América Latina y el Caribe (PREVAL); 2007. Biblioteca virtual. [Consultado: 29-12-13]. Disponible en: www.preval.org.

6. MEF, USAID y GIZ. Diagnóstico y propuesta de mejoras en la estrategia de diseño e implementación de un sistema de seguimiento y evaluación para el Perú: Ministerio de Economía y Finanzas; 2013.

7. Ministerio de Desarrollo e Inclusión Social (MIDIS) Conocer para incluir: Lineamientos para el Seguimiento, Evaluación, y Gestión de la Evidencia del MIDIS. Lima: Ministerio de Desarrollo e Inclusión Social (MIDIS). Dirección General de Seguimiento y Evaluación; 2012.
8. Ministerio de Salud (MINSA). Competencias Laborales para la Mejora del desempeño de Recursos Humanos en Salud. Lima: Ministerio de Salud (MINSA). Dirección General de Gestión del Desarrollo de Recursos Humanos; 2011.

9. Consejo de Evaluación, Acreditación y Certificación de la Calidad de la Educación Universitaria (CONEAU) [homepage en internet]. Lima: Certificación. Proceso de certificación. Entidades certificadoras Autorizadas DEC; c2013 [Consultado: 30-12-13]. Disponible en: http://www.coneau. gob.pe/certificacion/proceso-de-certificacion/ entidades-certificadoras-autorizadas.html.

10. Presidencia de Consejo de Ministros (PCM). Decreto Supremo que aprueba el "Plan Nacional de Desarrollo de Capacidades para la gestión Pública y Bien Gobierno de los Gobiernos regionales y Locales" DS 004-2010-PCM. El Peruano. 12 de enero de 2010.

11. Presidencia de Consejo de Ministros (PCM). Aprueban el Reglamento del Decreto Legislativo No. 1025 sobre Normas de Capacitación y Rendimiento para el Sector Público. DS 009-2010-PCM. El Peruano. 17 de enero de 2010.

12. Organización Internacional del Trabajo (OIT). Convenio 195. Recomendación sobre el desarrollo de los recursos humanos: educación, formación y aprendizaje permanente. Ginebra: OIT; 2005.

13. Vargas F. Competencias clave y aprendizaje permanente: tres miradas a su desarrollo en América Latina y el Caribe. Montevideo: Centro Interamericano para el Desarrollo del Conocimiento en la Formación Profesional (CINTEFOR)- Organización Internacional del Trabajo (OIT); 2004

14. Diploma Gestión de la Información de las Intervenciones Sanitarias. Lima; Julio 2010. Lima: Dirección General de Gestión del Desarrollo de Recursos Humanos-. Programa de Apoyo a la Reforma del Sector Salud II del Ministerio de Salud (MINSA); 2010.

15. USAID. Evaluation Policy. EVALUATION: Learning from Experience. Washington: USAID; 2011.

16. Soberon $\mathrm{L}$. Reunión técnica para la validación de competencias en monitoreo, evaluación y gestión de la evidencia de programas y proyectos de desarrollo organizado por USAID Evaluations. En: Evaluación de competencias profesionales. Lima: USAID Evaluations; 2013.

17. Alles M. Nuevo Enfoque Diccionario de Competencias La Trilogia. 60 Competencias más Utilizadas. Buenos Aires: Ed. GRANICA; 2012.

18. Alles M. Nuevo Enfoque Diccionario de Comportamientos La Trilogía. 1500 Comportamientos Relacionados con las Competencias más Utilizadas. Buenos Aires: Ed. GRANICA; 2012.

\section{Conflictos de interés:}

Los autores declaran no tener conflictos de interés.

\section{Correspondencia:}

Eva Miranda

San Borja Norte 552, San Borja, Lima - Perú

Correo electrónico: emiranda@pgrd.org 


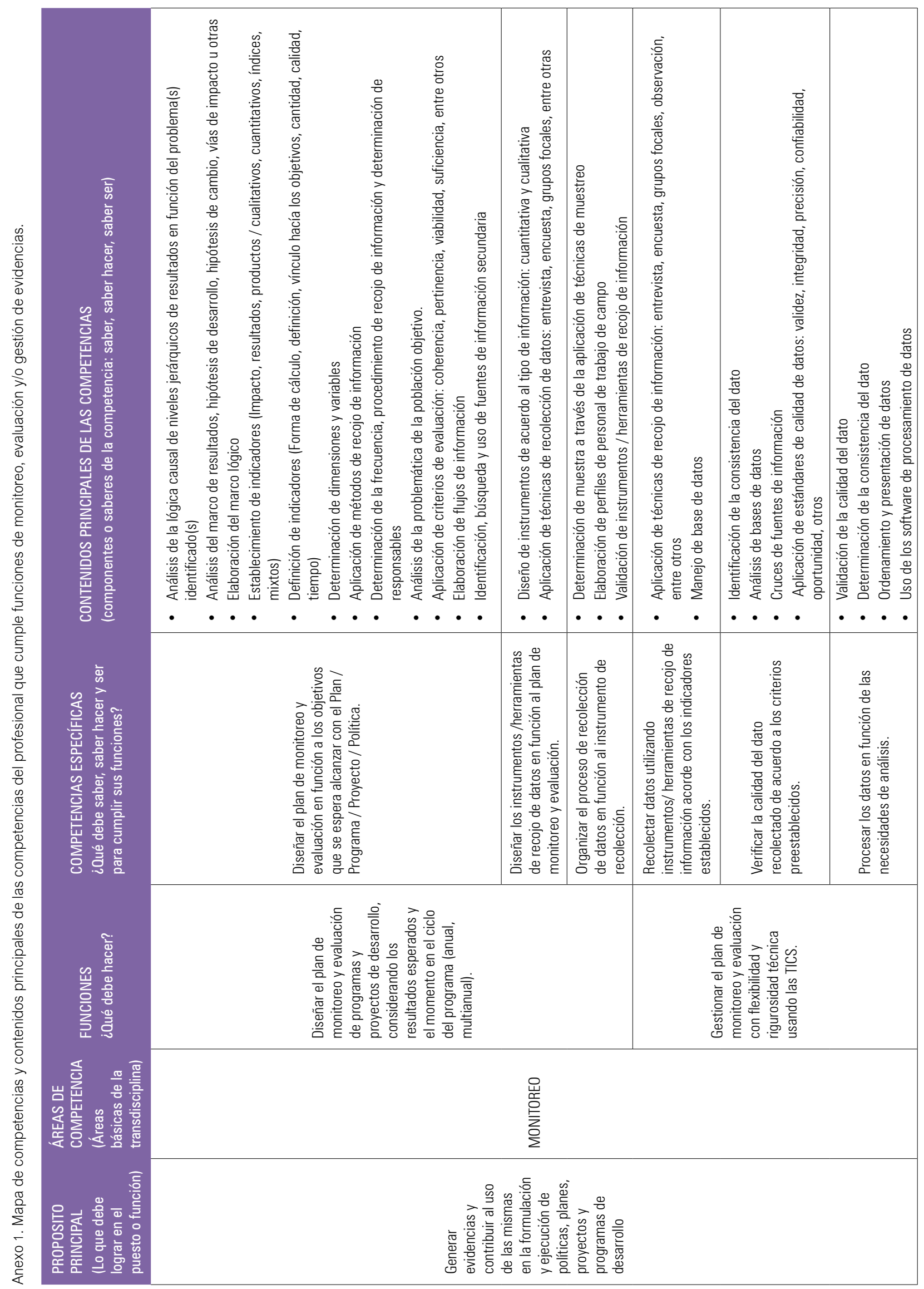




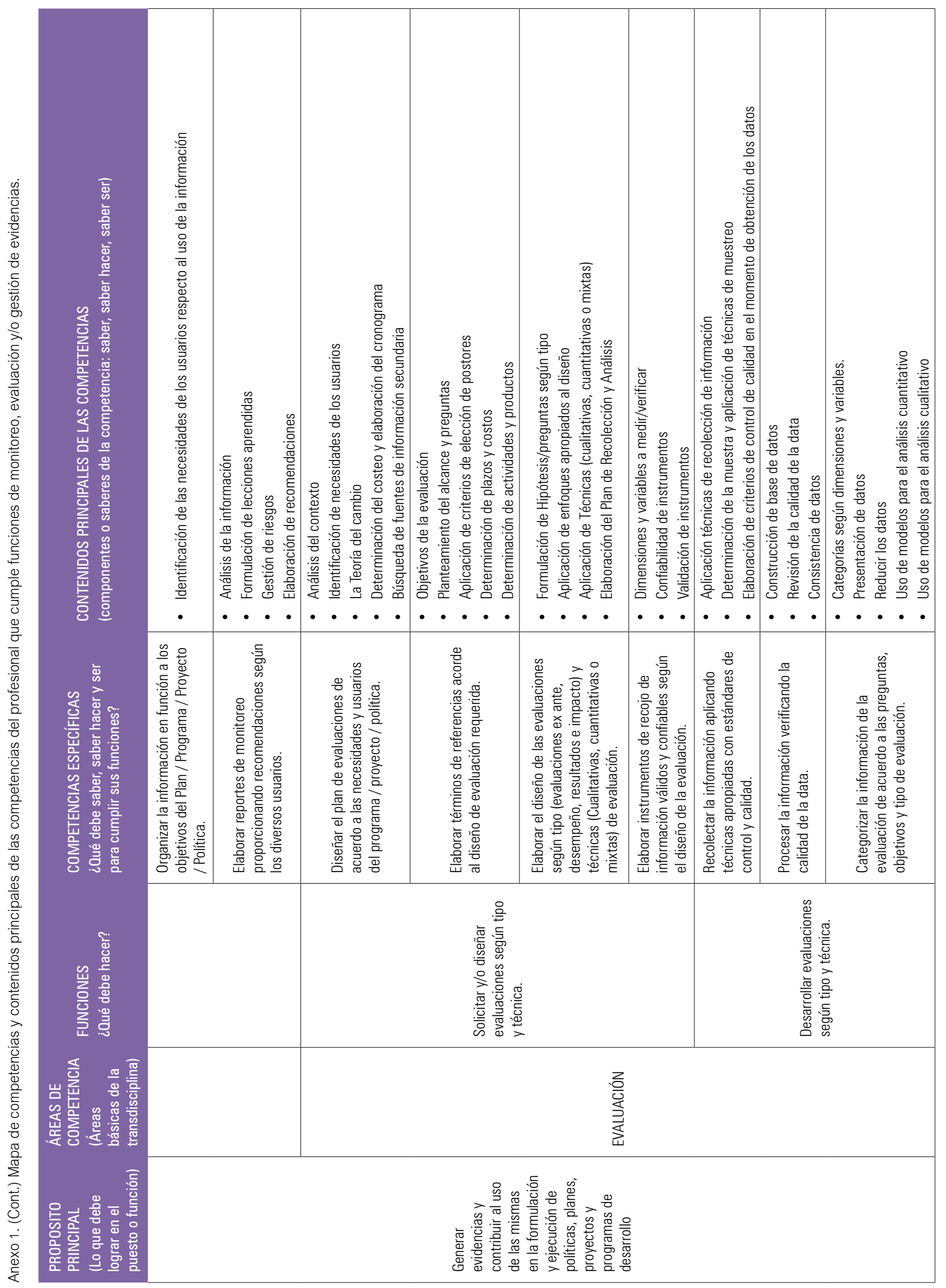




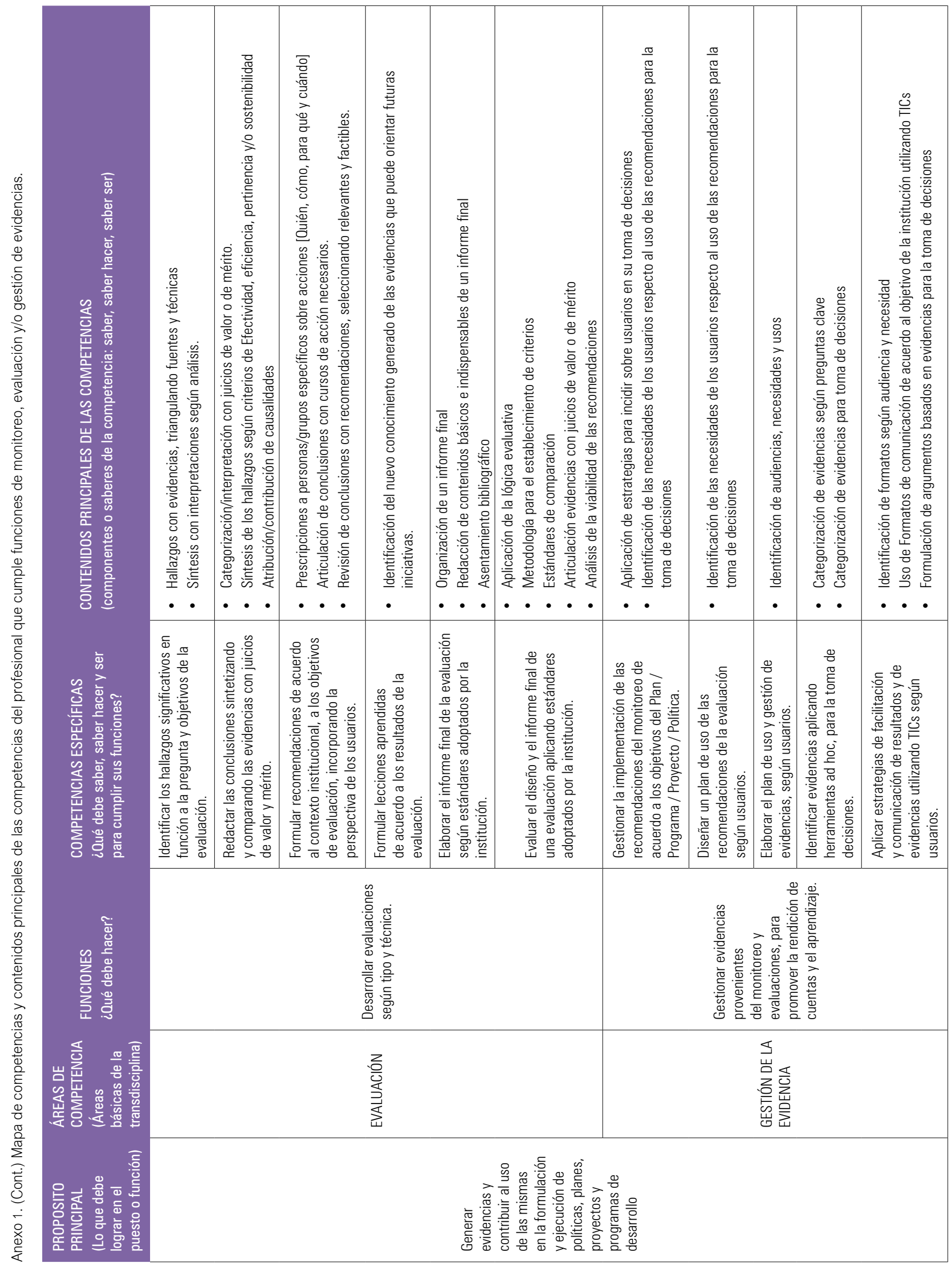

